\title{
SCYL1BP1 has Tumor-suppressive Functions in Human Lung Squamous Carcinoma Cells by Regulating Degradation of MDM2
}

\author{
Zhi-Ping Yang ${ }^{1}$, Yong-Hong Xie ${ }^{2}$, Dan-Yan Ling ${ }^{3}$, Jin-Rui Li ${ }^{1}$, Jin Jiang ${ }^{1}$, Yao- \\ Hua Fan ${ }^{1}$, Jia-Lian Zheng ${ }^{1}$, Wan-Xin Wu ${ }^{1 *}$
}

\begin{abstract}
SCY1-like 1-binding protein 1 (SCYL1BP1) is a newly identified transcriptional activator domain containing protein with many unknown biological functions. Recently emerging evidence has revealed that it is a novel regulator of the p53 pathway, which is very important for the development of human cancer. However, the effects of SCYL1BP1 on human lung squamous carcinoma cell biological behavior remain poorly understood. In this study, we present evidence that SCYL1BP1 can promote the degradation of MDM2 protein and further inhibit the G1/S transition of lung squamous carcinoma cell lines. Functional assays found that reintroduction of SCYL1BP1 into lung squamous carcinoma cell lines significantly inhibited cell proliferation, migration, invasion and tumor formation in nude mice, suggesting strong tumor suppressive function of SCYL1BP1 in lung squamous carcinoma. Taken together, our data suggest that the interaction of SCYL1BP1/MDM2 could accelerate MDM2 degradation, and may function as an important tumor suppressor in lung squamous carcinomas.
\end{abstract}

Keywords: SCYL1BP1 - MDM2 - lung squamous carcinoma cells - suppressor action

Asian Pac J Cancer Prev, 15 (17), 7467-7471

\section{Introduction}

Murine double minute 2 (MDM2) is involved in cell growth and differentiation through its interaction with other cellular proteins (Uldrijan et al., 2007; Wang et al., 2009). The MDM2 protein level is increased in a significant number of human tumors such as soft tissue sarcomas, osteosarcomas and breast tumors, underscoring its pivotal involvement in the development of human cancer (Cordon-Cardo et al., 1994; Liu et al., 2011; Ma et al., 2012; Wade et al., 2013).

MDM2 belongs to the family of RING Finger ubiquitin ligases (Jackson et al., 2000; Huang and Tindall, 2011), and the principal function of MDM2 is to mediate the ubiquitination and proteasome-dependent degradation of the p53 tumor suppressor protein and other growth regulatory proteins (Grossman et al., 1998; Manfredi, 2010; Chansaenroj et al., 2013). Accordingly, the ligase activity of MDM2 can be abolished by mutation of either one of eight cysteine and histidine residues involved in zinc coordination within the RING finger domain. Besides p53, MDM2 also ubiquitinates Numb (Yogosawa et al., 2003; Sczaniecka et al., 2012), $\beta$-arrestin binding protein (Shenoy et al., 2001; Kang et al., 2014), histone acetyltransferase Tip60 (Legube et al., 2002) as well as
MDM2 itself.

SCY1-like 1-binding protein 1 (SCYL1BP1) is a newly identified regulator of $\mathrm{Mdm} 2$, which directly binds to Mdm2 and promotes Mdm2 self-ubiquitination, subsequently stabilizing p53 (Yan et al., 2010a; Yan et al., 2010b). SCYL1BP1, initially identified as a SCYL1 binding protein (Di et al., 2003; Hu et al., 2012), is an evolutionally conserved protein, existing in different organisms ranging from plants to animals. SCYL1BP1 protein is observed in many normal tissues and mainly localized in the cytoplasm, with a low level in the nucleus. However, the interaction between SCYL1BP1 and MDM2 in human lung squamous carcinoma remained unknown, and the effects of SCYL1BP1 on lung squamous carcinoma cell biological behaviors still remains poorly understood.

In the pre sent study, we find that SCYL1BP1 could promote MDM2 degradation, which may through an ubiquitin-dependent pathway. Overexpression of SCYL1BP1 inhibited the G1/S transition in lung squamous carcinoma cell lines. Functional studies confirmed the tumor-inhibitory effect of SCYL1BP1 both in vitro and in vivo. These findings suggested that SCYL1BP1 may function as an important tumor suppressor gene in lung squamous carcinoma development. 


\section{Materials and Methods}

\section{Reagents and cell lines}

The following reagents and antibodies were used in this study: rabbit polyclonal anti-SCYL1BPI (Abcam), goat polyclonal anti-Mdm2 (Abcam), rabbit polyclonal anti- $\beta$-actin (Santa-Cruz, CA, USA), goat polyclonal anti-HA (Abcam). Z-Leu-Leu-Leu-al (MG132) and Cycloheximide (CHX) were purchased from Sigma Aldrich. The human lung squamous carcinoma cell lines (NCI-H226 and LTEP-S) were purchased from Cell Bank of Type Culture Collection of Chinese Academy of Sciences, Shanghai Institute of Cell Biology, and were passaged for fewer than 6 months. NCI-H226 and LTEP-S cells were cultured in RPMI 1640 medium (Gibco-BRL, Gaithersburg, MD, USA) supplemented with $10 \%$ fetal bovine serum (Gibco-BRL, Gaithersburg, MD, USA) and $1 \times$ antibiotics/antimycotics at $37^{\circ} \mathrm{C}$ in the presence of $5 \% \mathrm{CO}_{2}$.

\section{Plasmids, lentiviral production, and transduction}

The full-length cDNA of human SCYLIBP1 was synthesized by GeneWiz (Beijing, China). The fragment was digested by the XhoI and NotI (Fermentas, Hanover, MD, USA) incision enzymes and was cloned into the lentiviral expression vector pLVX-IRES-neo (Clonetech Laboratories Inc., San Francisco, CA) (Hwang-Verslues et al., 2011; Zhou and Wang, 2011; Li et al., 2014). The resulting construct (pLVX-IRES-neo-SCYL1BP) was verified by sequencing. Replication-defective VSV-G pseudotyped viral particles were packaged in the human embryonic kidney cells LentiX 293T (Clonetech Laboratories Inc.) by a three plasmid transient cotransfection method (Lenti-T HT packaging mix, Clontech) using plus Lipofectamine 2000 (Invitrogen) following the manufacturer's protocol. After $48 \mathrm{~h}$ of cotransfection, the lentivirus-containing supernatant was collected and passed through a $0.45-\mathrm{mm}$ filter. For transduction, NCI-H226 and LTEP-S cells were infected with control lentivirus (empty vector, only lentivirus without SCYL1BP was inserted) and SCYL1BP lentivirus, respectively. After $48 \mathrm{~h}$ of transduction, the cells were stably selected with G418 $(600 \mu \mathrm{g} / \mathrm{ml})$, and the drugresistant cell populations were used for subsequent studies.

\section{Quantitative real time PCR ( $q R T-P C R)$ analysis}

TRIzol reagent (Invitrogen) was used to extract total RNA from NCI-H226-control, NCI-H226-SCYL1BP1, LTEP-S-control, and LTEP-S-SCYL1BPI cells. An aliquot of total RNA $(1 \mu \mathrm{g})$ was reverse-transcribed into singlestrand complementary DNA using the M-MLV First Strand Kit (Invitrogen). The relative levels of RNA were detected using the ABI Prism 7000 sequence detection system (Applied Biosystems) with SYBR-Green Mix (Takara Bio, Otsu, Japan) and GAPDH as the internal control (Zheng et al., 2012). The primers used for MDM2 were 5'-TGA ACG ACA AAG AAAACG CCACAAAT3' and 5'-CAC TCT CCC CTG CCT GAT ACA CAG TA3' and for GAPDH were 5'-TTG GCC AGG GGT GCT AAG-3' and 5'-AGC CAAAAG GG TCA TCA TCTC-3' .

\section{Western blotting assay}

Extracts corresponding to cells were prepared by detergent lysis buffer. Sixty micrograms of total proteins was subjected to SDS-polyacrylamide gel electrophoresis (SDS-PAGE) and transferred to PVDF membrane (Millipore, Billerica, MA, USA). Antibodies for SCYL1BP1 (Abcam), MDM2 (Abcam), or $\beta$-actin (Santa-Cruz, CA, USA) were used. The membranes were incubated overnight at $4^{\circ} \mathrm{C}$ with the primary antibody diluted 1:1000 and the proteins were detected with a Phot otope-horseradish peroxidase Western blot detection kit (Cell Sigaling Technology, Danvers, MA, USA).

\section{Cell cycle analysis}

NCI-H226-control, NCI-H226-SCYL1BP1, LTEPS-control, and LTEP-S-SCYL1BP1 cells were plated at a concentration of $1 \times 10^{6}$ per $10 \mathrm{~cm}$ dish and fixed with chilled $70 \%$ ethanol overnight at $4^{\circ} \mathrm{C}$. Subsequently, we labeled single-cell suspensions with $50 \mu \mathrm{g} / \mathrm{ml}$ PI (Sigma, St Louis, MO) and analyzed approximately $1 \times 10^{6}$ cells in $1 \mathrm{ml}$ by flow cytometry. Using the FlowJo Software (Treestar, Inc, Ashland, OR), the percentage of cells in the $\mathrm{G} 2$ phase for 10,000 cells was denoted by the $2 \mathrm{~N}$ population phase.

\section{Cell viability assay}

NCI-H226-control, NCI-H226-SCYL1BP1, LTEP-Scontrol, and LTEP-S-SCYL1BP1 cells were collected and then seeded in 96-well flat-bottomed plates, and 2000 cells per $100 \mu \mathrm{l}$ of cell suspension were used to seed in each well. After 1,2, 4, and 6 days of cultivation, cell viability was measured by Cell Counting Kit-8 (CCK-8) system (Dojindo Laboratory, Kumamoto, Japan) according to the manufacturer's instructions. Briefly, CCK-8 solution $(10 \mu \mathrm{l}$ per $100 \mu \mathrm{l}$ of medium in each well) was added, the plates were then incubated at $37^{\circ} \mathrm{C}$ for $1 \mathrm{~h}$, and the absorbance of each well was read at $450 \mathrm{~nm}$ using a microplate reader (MRX; Dynex Technologies, West Sussex, United Kingdom). Six replicates for each group and the experiment were repeated at least 3 times.

\section{Xenografts in mice}

Female BALB/c nude mice, aged 4-5 weeks, were purchased from the Shanghai Laboratory Animal Center of the Chinese Academy of Sciences (Shanghai, China). Mice were allowed to acclimate to local conditions for at least 1 week and maintained under a $12 \mathrm{~h}$ dark, $12 \mathrm{~h}$ light cycle with food and water ad libitum. NCI-H226control, NCI-H226-SCYL1BP1, LTEP-S-control, and LTEP-S-SCYL1BP 1 cells were diluted to a concentration of $1 \times 10^{6} / \mathrm{mL}$ in physiological saline, Mice were injected subcutaneously with $0.1 \mathrm{ml}$ of the suspension into the back flank, each group consisted of 5 nude mice. When a tumor was palpable, tumor volume was measured by using a caliper every other day and calculated according to the following formula: $\mathrm{V}=\mathrm{L} \times \mathrm{W}^{2} \times 0.5$ (L, length; $\mathrm{W}$, width) (Zheng et al., 2013).

\section{Matrigel invasion assay}

Cell migration and invasion ability were determined by Corning transwell insert chambers with $8 \mu \mathrm{m}$ pores 
(Corning) and BD BioCoat Matrigel Invasion Chamber (Becton Dickinson Biosciences), respectively. About $2 \times 10^{4}$ (migration assay) or $2 \times 10^{5}$ (invasion assay) transfected cells in $200 \mu \mathrm{l}$ serum-free RPMI 1640 medium were seeded in the upper well and the chambers were incubated for $48 \mathrm{~h}$ at $37^{\circ} \mathrm{C}$ to allow the cells to migrate to the lower well with RPMI 1640 medium plus $20 \%$ fetal bovine serum. Cells that had migrated or invaded through the membrane were fixed with methanol and stained with crystal violet (Invitrogen), imaged and counted.

\section{Statistical analysis.}

All analyses were performed in a two-sided fashion using SAS (version 9.1; SAS Institute), and $p<0.05$ was defined as statistically significant. For comparisons, oneway analyses of variance, Fisher's exact tests, chi-squared tests, and two-tailed

Student's t-tests were performed, as appropriate. All data are presented as mean \pm SD. All experimental assays were performed in triplicated.

\section{Results}

SCYL1BP1 regulates MDM2 gene expression in human lung squamous carcinoma cells

To evaluate the effects of $S C Y L 1 B P 1$ on cell biological behaviors, we constructed lung squamous carcinoma cell lines with $S C Y L 1 B P 1$ stable overexpression and control group using lentiviruses (Figure 1A). Western blotting analysis showed that the expression of $M D M 2$ gene was diminished when $S C Y L 1 B P 1$ was overexpressed (Figure 1B), but we did not observe a reduction in MDM2 mRNA levels (Figure 1C).

To determine whether $S C Y L 1 B P 1$ reduced MDM2 protein stability, we treated $S C Y L 1 B P 1$-overexpressing and control NCI-H226 cells with the protein synthesis inhibitor, cycloheximide $(\mathrm{CHX})$, or the proteasome inhibitor MG-132. As shown in Figure 2A, only MG132 abolished the degradation of MDM2 protein levels in NCI-H226 cells overexpressing SCYL1BP1, and
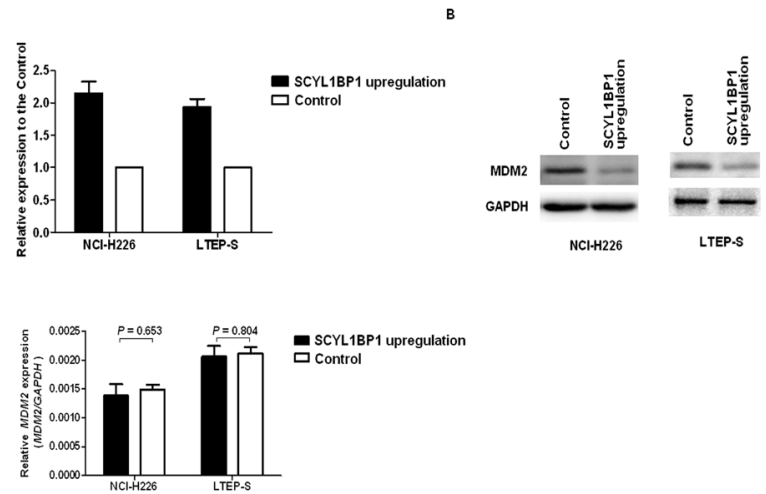

Figure 1. A) The $S C Y L 1 B P 1$ expression levels in lung squamous carcinoma cells after $S C Y L 1 B P 1$ or control lentiviruses infection. B) Western blot analysis for $M D M 2$ protein expression in $S C Y L 1 B P 1$ stable overexpressing and control cells. C) The relative mRNA levels of the MDM2 in $S C Y L 1 B P 1$ stable overexpressing, and control cell lines, data are mean \pm SEM, normalized to GAPDH overexpressing $S C Y L 1 B P 1$ promoted the degradation of $M D M 2$ protein levels Figure $2 \mathrm{~B}$. The same experiments were repeated using LTEP-S-control, and LTEP-S$S C Y L 1 B P 1$ cells with similar results (Figure 2Aand B).

SCYL1BP1 regulates the cell cycle in human lung squamous carcinoma cells.

We further investigated the effects of SCYL1BPI on cell cycle using fluorescence-activated cell sorting (FACS) analysis of propidium-iodide-stained lung squamous carcinoma cells. The representative results of the cellcycle distribution in empty vector, $S C Y L 1 B P 1$-transfected NCI-H226 and LTEP-S cells are shown in Figure 3A. Flow cytometry analysis revealed a statistically significant increase in the number of SCYL1BP1 overexpressed cells in the G1 phase (NCI-H226 cells: $11.08 \%$ increase, $p=0.0043$; LTEP-S cells: $8.93 \%$ increase, $p=0.0023$ ) accompanied by the decrease of S phase cells (NCI-H226 cells: $8.6 \%$ decrease, $p=0.0005$; LTEP-S cells: $11.48 \%$ decrease, $p=0.0007)$, compared to control-transfected cells (Figure 3A).

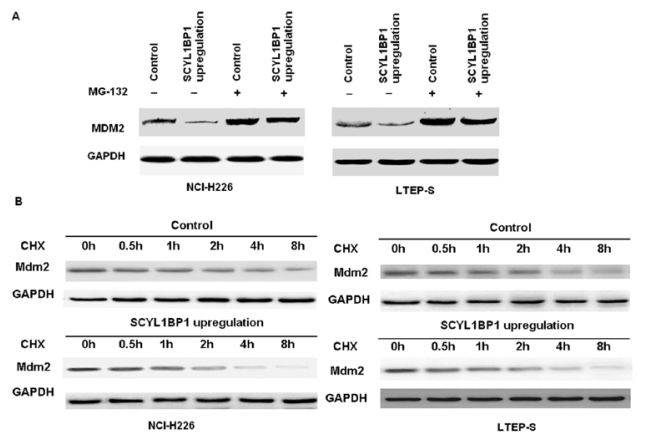

Figure 2. A and B) SCYL1BP1 stable overexpressing and control cells were incubated with the proteasome inhibitor MG-132 $(5 \mu M)$ or the protein synthesis inhibitor cycloheximide (CHX, $0.5 \mu \mathrm{g} / \mu \mathrm{l})$ for different time. The protein level of MDM2 in whole cell extracts was detected by western blotting

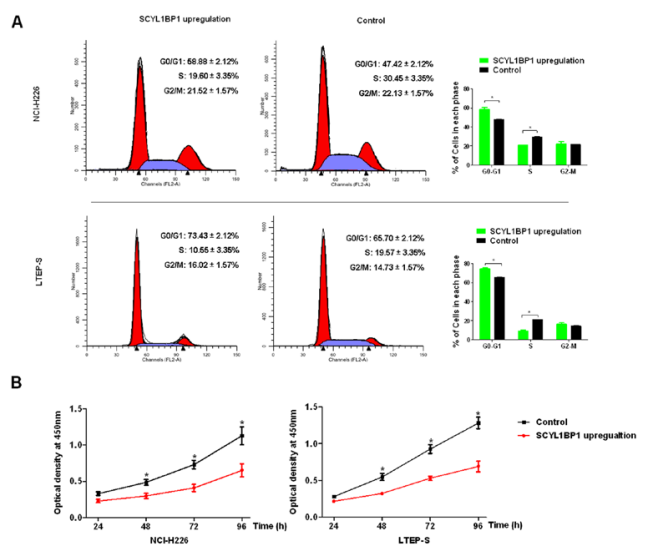

Figure 3. A) Cell cycle analysis of lung squamous carcinoma cells cells after transfected with SCYL1BP1 or Control lentiviruses. Results are represented as mean \pm SEM based on three independent experiments (*represents $p<0.05$ when compared to control). B) SCYL1BP1 stable overexpressing and control cells were seeded in 96-well plates, and cell proliferation was assessed daily for 4 days using the Cell Counting Kit-8 (CCK-8) assay (*represents $p<0.05$ when compared to control) 


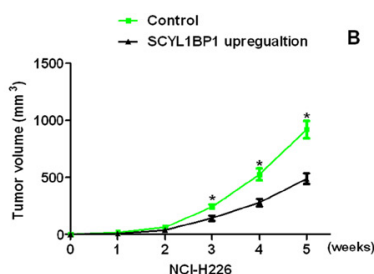

C

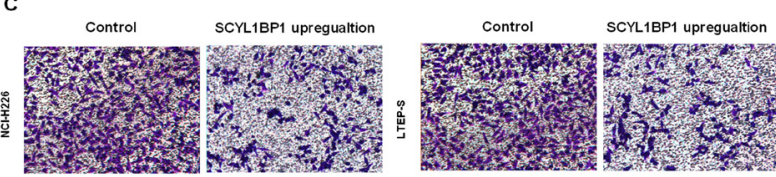

Figure 4. A) Subcutaneously implanted $S C Y L 1 B P 1$ stable overexpressing and control cells xenografted tumors were established. Bars, SEM. Mean tumor volume from 6 nude mice of each group are shown (*represents $p<0.05$ when compared to control). B) Matrigel invasion assays were performed in 24-well transwell chambers containing polycarbonate filters with $8 \mu \mathrm{m}$ pores coated with matrigel by using the SCYL1BP1 stable overexpressing and control cells. Results are represented as mean \pm SEM based on three independent experiments

Effects of SCYL1BPI on human lung squamous carcinoma cells proliferation

Cell counting kit-8 (CCK-8) assays indicated that the cell proliferation, assessed daily for 4 days, was consistently suppressed in NCI-H226 (optical density [OD], $0.65 \pm 0.09$ vs. $1.13 \pm 0.12, p=0.011)$ and LTEP-S cells $(0.69 \pm 0.07 v s .1 .28 \pm 0.08, p=0.0002)$ with exogenous expression of $S C Y L 1 B P 1$, compared to the control cells on the fourth day (Figure 3B).

\section{Effects of SCYL1BP1 on tumor growth}

To probe the effects of SCYL1BP1 on cancer cell dynamics in vivo, SCYL1BP1-upregulated (NCI-H226SCYL1BP1 and LTEP-S- SCYL1BP1 cells), and respective control cells were injected into the back flank of nude mice. Our results showed that the growth of tumors from SCYL1BP1-upregulated xenografts was significantly inhibited when compared with that of tumors formed from control xenografts: $488.2 \pm 47.26 \mathrm{~mm} 3$ versus $919.9 \pm 75.73 \mathrm{~mm} 3$ of NCI-H226 cells ( $p=0.0013$ ); $585.4 \pm 54.85 \mathrm{~mm} 3$ versus $921.9 \pm 80.04 \mathrm{~mm} 3$ of LTEP-S cells $(p=0.0085)$, respectively (Figure 4A and 4B).

\section{Effects of SCYL1BPI on human lung squamous carcinoma} cells invasion

Using transwell chambers precoated with or without Matrigel, we examined the effect of SCYL1BP1 on cell invasion. Cells with higher expression of SCYL1BP1 (both NCI-H226 and LTEP-S cell lines) showed significantly decreased migration and invasion when compared to the control cells (Figure 4C).

\section{Discussion}

SCYL1BP1 was initially identified as an SCYL1binding protein (Di et al., 2003; Liu et al., 2012), but little is known about its biological function in human lung squamous carcinoma. Previous study showed that loss-of-function mutations of SCYL1BP1 caused gerodermia osteodysplastica (Hennies et al., 2008; Bexiga and Simpson, 2013), which suggested that SCYL1BP1 is an important pathogenic candidate gene. More recently, SCYL1BP1 was identified as a new regulator of the p53 pathway (Yan et al., 2010a). So it is perhaps not surprising that SCYL1BP1 has broader roles in physiology and pathology, such as the regulation of stress, metabolism, autophagy, tumors, and development. Here our specific aim is to investigate the potential roles of SCYL1BP1 in the development of lung squamous carcinoma.

In the present study, we found SCYL1BP1 can promote the degradation of MDM2 in lung squamous carcinoma cell lines. Overexpression of SCYL1BP1 decreased the expression of MDM2 at protein level but not at mRNA level. Protease inhibitor MG132 inhibited the degradation of MDM2 in SCYL1BP1 overexpressing cells. Recently, SCYL1BP1 was found to accelerate the self-ubiquitination of MDM2 and suppress the MDM2mediated p53 degradation (Momand et al., 1992; Yan et al., 2010b). Our result further confirmed this finding in lung squamous carcinoma. MDM2 is an important E3 ligase that may regulate the proteasomal degradation of p53 at different circumstances (Brooks and Gu, 2006; Sparks et al., 2013). It is possible that SCYL1BP1 may regulate the stability of p53 through this pathway. SCYL1BP1 may be a potential critical protein involved in the $\mathrm{p} 53$ pathway, for it can interact with and regulate the stability of MDM2. Since p53 is an important regulator of G1/S cell cycle check point (Martinez et al., 1991; Polyak et al., 1994; van der Laan et al., 2013), we next examined the effect of SCYL1BP1 on cell cycle progression. As expected, cell cycle analysis found that SCYL1BP1 could arrest cell cycle at G1/S checkpoint in NCI-H226 and LTEP-S cells. These data suggested that SCYL1BP1 may regulate cell cycle arrest mainly through the p53 pathway. To further explore the tumor-suppressive function of SCYL1BP1 in lung squamous carcinoma development, SCYL1BP1 was transfected into two lung squamous carcinoma cell lines. Functional studies found that the ectopic expression of SCYL1BP1 in NCI-H226 and LTEP-S cells dramatically inhibited cell growth, migration, invasion and tumor formation in nude mice, indicating that SCYL1BP1 had strong tumor-suppressive function during lung squamous carcinoma progression.

Based on these findings, we propose that SCYL1BP1 is a novel regulator of MDM2 stability in lung squamous carcinoma. Ectopic expression enhanced the degradation of MDM2and inhibited the G1/S transition of lung squamous carcinoma cell lines and exhibited strong tumorsuppressive function both in vitro and in vivo. The frequent downregulation may account for the overexpression of MDM2 in the development of lung squamous carcinoma. Further characterization of the tumor-suppressive function of SCYL1BP1 may greatly facilitate our understanding of the molecular mechanism of lung squamous carcinoma development and find new thera peutic targets for lung squamous carcinoma treatment.

\section{Acknowledgements}

This work was supported by the Science and Technology Project of Zhejiang province (No. 2013C33107). 


\section{References}

Bexiga MG, Simpson JC (2013). Human diseases associated with form and function of the Golgi complex. Int $\mathrm{J} \mathrm{Mol}$ Sci, 14, 18670-81.

Brooks CL, Gu W (2006). p53 ubiquitination: Mdm2 and beyond. Mol Cell, 21, 307-15.

Chansaenroj J, Theamboonlers A, Junyangdikul P, et al (2013). Polymorphisms in TP53 (rs1042522), p16 (rs11515 and rs3088440) and NQO1 (rs1800566) genes in Thai cervical cancer patients with HPV 16 infection. Asian Pac J Cancer Prev, 14, 341-6.

Cordon-Cardo C, Latres E, Drobnjak M, et al (1994). Molecular abnormalities of mdm2 and 553 genes in adult soft tissue sarcomas. Cancer Res, 54, 794-9.

Di Y, Li J, Fang J, et al (2003). Cloning and characterization of a novel gene which encodes a protein interacting with the mitosis-associated kinase-like protein NTKL. J Hum Genet, 48, 315-21.

Grossman SR, Perez M, Kung AL, et al (1998). p300/MDM2 complexes participate in MDM2-mediated p 53 degradation. Mol Cell, 2, 405-15.

Hennies HC, Kornak U, Zhang H, et al (2008). Gerodermia osteodysplastica is caused by mutations in SCYL1BP1, a Rab-6 interacting golgin. Nat Genet, 40, 1410-2.

$\mathrm{Hu}$ L, Liu M, Chen L, et al (2012). SCYL1 binding protein 1 promotes the ubiquitin-dependent degradation of Pirh2 and has tumor-suppressive function in the development of hepatocellular carcinoma. Carcinogenesis, 33, 1581-8.

Huang H, Tindall DJ (2011). Regulation of FOXO protein stability via ubiquitination and proteasome degradation. Biochim Biophys Acta, 1813, 1961-4.

Hwang-Verslues WW, Chang PH, Wei PC, et al (2011). miR495 is upregulated by E12/E47 in breast cancer stem cells, and promotes oncogenesis and hypoxia resistance via downregulation of E-cadherin and REDD1. Oncogene, 30, 2463-74.

Jackson PK, Eldridge AG, Freed E, et al (2000). The lore of the RINGs: substrate recognition and catalysis by ubiquitin ligases. Trends Cell Biol, 10, 429-39.

Kang DS, Tian X, Benovic JL (2014). Role of beta-arrestins and arrestin domain-containing proteins in $\mathrm{G}$ protein-coupled receptor trafficking. Curr Opin Cell Biol, 27, 63-71.

Legube G, Linares LK, Lemercier C, et al (2002). Tip60 is targeted to proteasome-mediated degradation by $\mathrm{Mdm} 2$ and accumulates after UV irradiation. EMBO J, 21, 1704-12.

Li W, Zheng J, Deng J, et al (2014). Increased levels of the long intergenic non-protein coding RNAPOU $3 \mathrm{~F} 3$ promote DNA methylation in esophageal squamous cell carcinoma cells. Gastroenterology, 146, 1714-26

Liu J, Zheng Y, Lei D, et al (2011). MDM2 309T>G polymorphism and risk of squamous cell carcinomas of head and neck: a meta-analysis. Asian Pac J Cancer Prev, 12, 1899-903.

Liu Y, Chen Y, Lu X, et al (2012). SCYL1BP1 modulates neurite outgrowth and regeneration by regulating the $\mathrm{Mdm} 2 / \mathrm{p} 53$ pathway. Mol Biol Cell, 23, 4506-14.

Ma HB, Huang T, Han F, et al (2012). Association between MDM2 promoter SNP309 T/G polymorphism and liver cancer risk - a meta-analysis. Asian Pac J Cancer Prev, 13, 2841-6.

Manfredi JJ (2010). The Mdm2-p53 relationship evolves: Mdm2 swings both ways as an oncogene and a tumor suppressor. Genes Dev, 24, 1580-9.

Martinez J, Georgoff I, Levine AJ (1991). Cellular localization and cell cycle regulation by a temperature-sensitive p53 protein. Genes Dev, 5, 151-9.
Momand J, Zambetti GP, Olson DC, et al (1992). The mdm-2 oncogene product forms a complex with the $\mathrm{p} 53$ protein and inhibits p53-mediated transactivation. Cell, 69, 1237-45.

Polyak K, Lee MH, Erdjument-Bromage H, et al (1994). Cloning of p27Kip1, a cyclin-dependent kinase inhibitor and a potential mediator of extracellular antimitogenic signals. Cell, 78, 59-66.

Sczaniecka M, Gladstone K, Pettersson S, et al (2012). $M D M 2$ protein-mediated ubiquitination of numb protein: identification of a second physiological substrate of $M D M 2$ that employs a dual-site docking mechanism. J Biol Chem, 287, 14052-68.

Shenoy SK, McDonald PH, Kohout TA, et al (2001). Regulation of receptor fate by ubiquitination of activated beta 2 -adrenergic receptor and beta-arrestin. Science, 294, 1307-13.

Sparks A, Dayal S, Das J, et al (2013). The degradation of p53 and its major E3 ligase Mdm2 is differentially dependent on the proteasomal ubiquitin receptor S5a. Oncogene, [Epub ahead of print].

Uldrijan S, Pannekoek WJ, Vousden KH (2007). An essential function of the extreme C-terminus of $M D M 2$ can be provided by MDMX. EMBO J, 26, 102-12.

van der Laan S, Tsanov N, Crozet C, et al (2013). High Dub3 expression in mouse ESCs couples the G1/S checkpoint to pluripotency. Mol Cell, 52, 366-79.

Wade M, Li YC, Wahl GM (2013). MDM2, MDMX and p53 in oncogenesis and cancer therapy. Nat Rev Cancer, 13, 83-96.

Wang SP, Wang WL, Chang YL, et al (2009). p53 controls cancer cell invasion by inducing the MDM2-mediated degradation of Slug. Nat Cell Biol, 11, 694-704.

Yan J, Di Y, Shi H, et al (2010a). Overexpression of SCYL1-BP1 stabilizes functional p53 by suppressing MDM2-mediated ubiquitination. FEBS Lett, 584, 4319-24.

Yan J, Zhang D, Di Y, et al (2010b). A newly identified Pirh2 substrate SCYL1-BP1 can bind to MDM2 and accelerate MDM2 self-ubiquitination. FEBS Lett, 584, 3275-8.

Yogosawa S, Miyauchi Y, Honda R, et al (2003). Mammalian Numb is a target protein of Mdm2, ubiquitin ligase. Biochem Biophys Res Commun, 302, 869-72.

Zheng J, Deng J, Xiao M, et al (2013). A sequence polymorphism in miR-608 predicts recurrence after radiotherapy for nasopharyngeal carcinoma. Cancer Res, 73, 5151-62.

Zheng J, Jiang L, Zhang L, et al (2012). Functional genetic variations in the IL-23 receptor gene are associated with risk of breast, lung and nasopharyngeal cancer in Chinese populations. Carcinogenesis, 33, 2409-16.

Zhou J, Wang W (2011). Analysis of microRNA expression profiling identifies microRNA-503 regulates metastatic function in hepatocellular cancer cell. J Surg Oncol, 104, 278-83. 